\title{
Thermal Impulse Response in Porous Media for Transpiration Cooling Systems
}

\author{
Tobias Hermann *, Matthew McGilvray ${ }^{\dagger}$, Hassan Saad Ifti \\ The University of Oxford, Department of Engineering, Osney Mead, Southwell Building, Oxford OX2 OES, United \\ Kingdom \\ Fabian Hufgard ${ }^{\S}$ and Stefan Löhle ${ }^{\mathbb{I l}}$ \\ Universität Stuttgart, Institut für Raumfahrtsysteme, Pfaffenwaldring 29, D-70569 Stuttgart, Germany
}

A solution of the coupled differential equations for fluid and solid phase in a one-dimensional porous medium in thermal non-equilibrium is presented using the concept of analyzing the impulse response. The impulse response is shown to be sensitive to the volumetric heat transfer coefficient and the coolant mass flux. Experimental data obtained from surface heating of transpiration cooled porous $\mathrm{ZrB}_{2}$ samples is compared to a newly developed theoretical model. The surface and backside temperature of the solid are measured using thermographic imaging and thermocouple instrumentation. The non-integer system identification approach is used to experimentally obtain the thermal impulse response which is then compared to the model prediction. Good agreement is found between the simulated and experimental data with average deviations below $10 \%$. The developed model provides the basis for inverse heat transfer measurements and further analysis of transpiration cooled materials.

\section{Nomenclature}

$a \quad$ Heat loss constant, $\mathrm{W} \mathrm{m}^{-3} \mathrm{~K}^{-1}$

A Coefficient in Laplace-space

B Coefficient in Laplace-space

$c_{p} \quad$ Specific heat capacity, $\mathrm{J} \mathrm{kg}^{-1} \mathrm{~K}^{-1}$

C Coefficient for analytical temperature solution, $\mathrm{K}$

$d \quad$ Length of square illuminated by the laser radiation

$H \quad$ Impulse response, $\mathrm{K}$

$h_{v} \quad$ Volumetric heat transfer coefficient, $\mathrm{W} \mathrm{m}^{-3} \mathrm{~K}^{-1}$

$J \quad$ Iteration parameter

\footnotetext{
* Post-doctoral research assistant, Hypersonics group, Oxford Thermofluids Institute, AIAA Member.

$\dagger$ Associate Professor, Hypersonics group, Oxford Thermofluids Institute, AIAA Member.

DPhil student, Hypersonics group, Oxford Thermofluids Institute, AIAA Member.

${ }^{\S}$ PhD student, High Enthalpy Flow Diagnostics Group, Institute of Space Systems.

"Research engineer, Head of High Enthalpy Flow Diagnostics Group, Institute of Space Systems.
} 
$k \quad$ Thermal conductivity, $\mathrm{W} \mathrm{m}^{-1} \mathrm{~K}^{-1}$

$K_{D} \quad$ Darcy coefficient, $\mathrm{m}^{2}$

$K_{F} \quad$ Forchheimer coefficient, $\mathrm{m}$

$L \quad$ Thickness, $\mathrm{m}$

M Iteration parameter

$m \quad$ Mass, $\mathrm{kg}$

$m \quad$ Iteration parameter

$N \quad$ Iteration parameter

$n \quad$ Iteration parameter

$\dot{m} \quad$ Mass flow, $\mathrm{kg} \mathrm{s}^{-1}$

$\dot{m}_{A} \quad$ Mass flux, $\mathrm{kg} \mathrm{m}^{-2} \mathrm{~s}^{-1}$

$\dot{q} \quad$ Heat flux, $\mathrm{W} \mathrm{m}^{-2}$

$\tilde{q} \quad$ Laplace transformed heat flux, $\mathrm{W} \mathrm{m}^{-2}$

$\dot{q}_{L} \quad$ Heat loss, $\mathrm{W} \mathrm{m}^{-3}$

$P_{\text {laser }}$ Laser power, $\mathrm{W}$

$s \quad$ Laplace variable

$t$ Time, $\mathrm{s}$

$T \quad$ Temperature, $\mathrm{K}$

$\tilde{T} \quad$ Laplace transformed temperature, $\mathrm{K}$

$u_{m} \quad$ Bulk velocity of coolant, $\mathrm{m} \mathrm{s}^{-1}$

$V_{n} \quad$ Gaver-Stehfest coefficient

$x \quad$ Spatial variable, $\mathrm{m}$

$\alpha \quad$ Thermal diffusivity, $\mathrm{m}^{2} \mathrm{~s}^{-1}$

$\alpha_{m} \quad$ NISI-coefficient for temperature

$\beta_{n} \quad$ NISI-coefficient for heat flux

$\delta \quad$ Dirac impulse, $\mathrm{s}^{-1}$

$\varepsilon \quad$ Surface emissivity

$\lambda \quad$ Eigenvalue, $\mathrm{m}^{-1}$

$\phi \quad$ Porosity

$\rho \quad$ Density, $\mathrm{kg} \mathrm{m}^{-3}$

$\tau \quad$ Time in convolution integral, $\mathrm{s}$

Subscript 


$\begin{array}{ll}0 & \text { starting parameter } \\ \text { abs } & \text { absolute } \\ f & \text { fluid } \\ i & \text { iteration parameter } \\ n & \text { iteration parameter } \\ & \\ s & \text { solid }\end{array}$

\section{Introduction}

Actively cooled porous thermal protection systems have potential applications in future hypersonic vehicles that are exposed to heat fluxes which cannot be managed by passive cooling [1-7]. Thermal management of wall temperatures and absorbed heat load of re-entry vehicles or hypersonic cruise vehicles is one of the major challenges of such systems [8]. Porous media are envisaged to enable comparably simple transpiration cooled systems, where relatively cool fluid is fed through a porous material at the outer face [9-13]. There are two primary cooling mechanisms: Firstly, the gas exiting the porous wall forms a coolant layer between hot external flow and the wall which reduces the aerodynamic heat load experienced by the vehicle. This effect leads to lower boundary layer temperatures and inhibits surface recombination and oxidation reactions [14,15]. Secondly, the convective heat exchange between the hot solid

phase and the colder fluid phase within the porous medium leads to the cooling of the entire wall segment [16-19]. The maximum performance occurs when the coolant exits the porous wall at the surface temperature, i. e. at a local thermal equilibrium condition. In this case, the coolant has extracted the maximum heat from the wall. The design of a transpiration cooled thermal protection system is challenging as the internal heat transfer, the pressure loss over the porous wall, the internal temperature field, and the coolant mass flow rate are coupled.

In order to assess the performance of transpiration cooling, measurements are required that quantify both the internal heat transfer due to convection and the external effect due to the generation of a protective film. The measurement of both components is accessible through the thermal impulse response of the porous medium. This paper presents the theoretical derivation and methodology to determine the impulse response. In the following, a short review of this concept is given and then its application to determine the internal and external cooling components is discussed.

An impulse response $H(t, x)$ describes the transient temperature trace at a certain location $x$ within a material which has been subject to a heat flux impulse at the surface. The impulse response of a particular linear thermal system, i. e. constant thermophysical parameters, is unique and hence describes its thermal behaviour completely [20]. The heat flux is defined as a Dirac impulse at time $t=0$ which has an amplitude of infinity and an integral of $1 \mathrm{~J}^{-2}$. An example of a surface temperature impulse response of a semi-infinite problem is shown in Fig. 11. In this case, the 


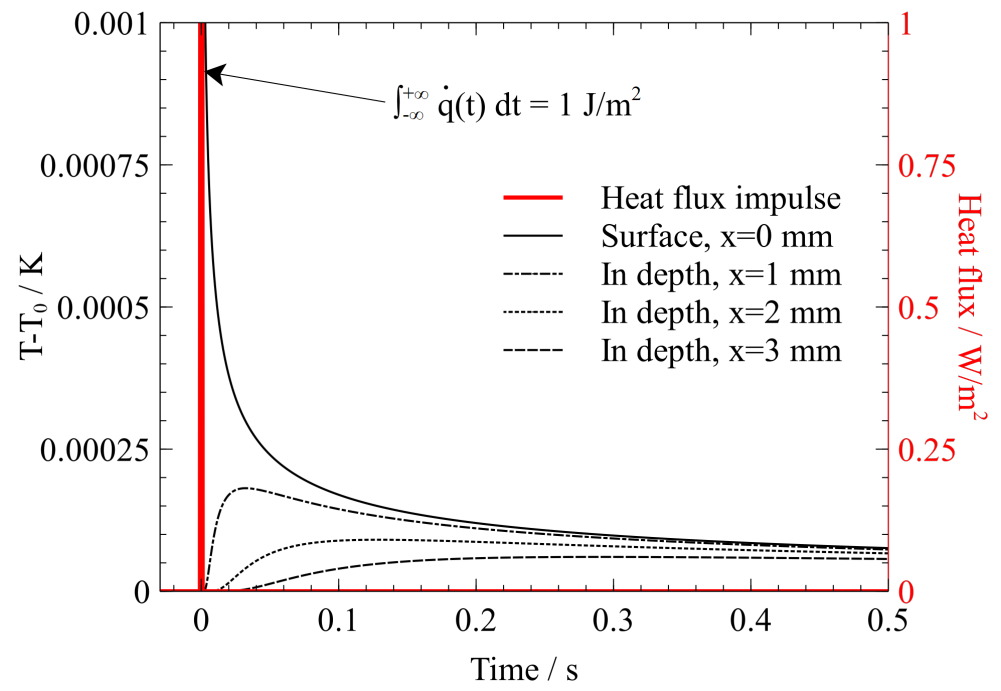

Fig. 1 Example of a heat flux impulse and the respective surface temperature impulse response for a semiinfinite one-dimensional case.

analytical solution for a purely solid material is

$$
T(t, x=0)=H(t, x=0)=\frac{1}{\sqrt{\rho c_{p} k}} \sqrt{\frac{1}{\pi t}}
$$

with temperature, $T$, spatial location, $x$, time, $t$, density, $\rho$, specific heat capacity, $c_{p}$, and thermal conductivity, $k$ [21]. Figure 1 also contains impulse responses at various depths $x$ within the material which are calculated via

$$
T(t, x)=H(t, x)=\sqrt{\frac{\alpha}{\pi t k^{2}}} \exp \left(\frac{-x^{2}}{4 \alpha t}\right)
$$

with the thermal diffusivity $\alpha=k /\left(\rho c_{p}\right)$ [22].

Impulse responses are a versatile tool for the analysis of inverse heat conduction problems. The most popular application is the determination of the transient surface heat transfer from a temperature measurement [20]. The convolution of surface heat flux $\dot{q}(t, x=0)$ and impulse response $H(t, x)$ results in the respective sensor temperature

$$
T(t, x)=H(t, x) * \dot{q}(t, x=0)=\int_{-\infty}^{\infty} H(\tau, x) \dot{q}(t-\tau, x=0) \mathrm{d} \tau
$$

at a location $x$ in the material. Equation (3) is usually deconvolved using some regularization scheme to obtain the heat flux in the convolution integral [23, 24]. Hence, in order to measure the net heat flux to a porous material the respective impulse response is required. For a transpiration cooled wall segment, the net heat flux is some fraction of the original aerodynamic heat flux. The presence of the external film leads to a reduction of this heat flux and hence constitutes 
one of the aforementioned cooling mechanism. These experiments are challenging, as the analysis of heat transfer in a porous medium is complicated by the internal coolant flow which influences the impulse response. Therefore, an alternative theoretical description of the impulse response is required and conventional equations, e. g. Eqs. (1) and (2), cannot be applied.

In addition to using the impulse response as a means to determine the external cooling effectiveness it can also be used to assess the internal heat transfer characteristics. As will be shown in the current work, the thermal impulse response of a porous medium is significantly influenced by the internal heat transfer of a porous material. When internal cooling is more effective, more heat will be transferred from solid to fluid phase. This effect will also be apparent as the impulse response of the solid material will change in magnitude and shape. Through the development of the theoretical framework, this effect can be exploited to infer the magnitude of the internal heat transfer, usually expressed through the volumetric heat transfer coefficient $h_{v}$, from the measurement of the impulse response.

As mentioned above, the impulse response can be used to enable cooling effectiveness measurements and material characterization. This paper derives the approach that allows the determination of the impulse response of fluid and solid phase for a transpiration cooled problem and provides a comparison to experimental data using a laser calibration approach. The mathematical derivation will be presented first and then the methodology for the experimental verification will be discussed. A comparison between the impulse responses determined with the newly developed model and those determined experimentally is presented. Furthermore, the effect of varying the local thermal non-equilibrium between the two phases in the material will be investigated for the application to an actively cooled thermal protection system.

\section{Theory}

The coupled partial differential energy equations of a porous medium are solved to deduce the temperature response of solid and fluid phase which can be in thermal non-equilibrium due to a unit impulse of heat at the surface. The one-dimensional energy equation for the solid phase is

$$
\underbrace{(1-\phi) \rho_{s} c_{p, s} \frac{\partial T_{s}}{\partial t}}_{\text {Transient heating (solid) }}=\underbrace{k_{s}(1-\phi) \frac{\partial^{2} T_{s}}{\partial x^{2}}}_{\text {In depth heat conduction (solid) }}+\underbrace{\dot{q}_{L}}_{\text {Lateral heat conduction }}+\underbrace{h_{v}\left(T_{f}-T_{s}\right)}_{\text {Fluid solid heat exchange }}
$$

and the respective energy equation for the fluid phase is

$$
\underbrace{\phi \rho_{f} c_{p, f} \frac{\partial T_{f}}{\partial t}}_{\text {Transient heating (fluid) }}=\underbrace{\phi \rho_{f} c_{p, f} \frac{u_{m}}{\phi} \frac{\partial T_{f}}{\partial x}}_{\text {Convection }}+\underbrace{h_{v}\left(T_{s}-T_{f}\right)}_{\text {Solid fluid heat exchange }}
$$

with porosity, $\phi$, bulk velocity, $u_{m}$, heat loss term, $\dot{q}_{L}$, temperature difference, $T=\left(T_{\mathrm{abs}}-T_{0}\right)$ and $s$ denoting solid and $f$ denoting fluid [25]. Here, $T_{0}$ is the starting temperature and $T_{\text {abs }}$ is the absolute temperature. The inclusion of a heat 
loss term $\dot{q}_{L}$ represents the inevitable lateral heat conduction in a non-adiabatic experimental setup, an approach which has previously been used by Langener [9]. The lateral heat loss is assumed to be dominated by conduction in the solid phase, since the thermal conductivity of the fluid phase is negligibly small. The small thermal conductivity of the fluid is also the reason for omitting any conduction terms in Eq. (5). The heat loss term is assumed to be of the form

$$
\dot{q}_{L}=a T_{s}
$$

with the constant $a$ to be empirically determined from the particular experiment. This formulation assumes a constant temperature of the mount containing the porous piece (see section III.A for details of the experimental setup that is used to validate the developed model). The reason for this assumption is that the thermal mass $\left(c_{p} \cdot m\right)$ of the surrounding experiment is 55 times larger compared to the thermal mass of the heated porous sample. Hence, heat conducted into the outer structure will not lead to a significant increase in its temperature. Therefore, the lateral conduction will be mostly driven by the solid temperature which is reflected in Eq. (6).

The corresponding boundary conditions for Equations (4) and (5) are

$$
\begin{gathered}
\underbrace{T_{f}(t>0, x=L)=0,}_{\text {Constant fluid temperature at backside }} \\
\underbrace{\frac{\partial T_{s}}{\partial x}(t>0, x=L)=0}_{\text {Adiabatic backside }}, \\
\underbrace{\dot{q}(t>0, x=0)=-(1-\phi) k_{s} \frac{\partial T_{s}}{\partial x}(t>0, x=0)=\delta(t=0)}_{\text {Heat Impulse on surface }},
\end{gathered}
$$

with thickness $L$ and Dirac impulse $\delta(t)$. For the solution of Eq. (4) and (5) it is assumed that the thermophyscial parameters $\rho, c_{p}$ and $k$ of both solid and fluid phase are not a function of temperature and constant throughout the material.

The solution of the coupled partial differential equations is achieved by applying a Laplace transform with respect to the time dimension and assuming an isothermal material at $T=0$ before the heat impulse is applied. Eq. (4) and (5) reduce to

$$
s(1-\phi) \rho_{s} c_{p, s} \tilde{T}_{s}=k_{s}(1-\phi) \frac{\partial^{2} \tilde{T}_{s}}{\partial x^{2}}+h_{v}\left(\tilde{T}_{f}-\tilde{T}_{s}\right)+a \tilde{T}_{s}
$$

and

$$
s \phi \rho_{f} c_{p, f} \tilde{T}_{f}=\phi \rho_{f} c_{p, f} \frac{u_{m}}{\phi} \frac{\partial \tilde{T}_{f}}{\partial x}+h_{v}\left(\tilde{T}_{s}-\tilde{T}_{f}\right)
$$


with the Laplace variable $s$ and the Laplace-transformed temperature $\tilde{T}$. Rearranging Eq. 10 results in

$$
\tilde{T}_{f}=\left(\frac{s(1-\phi) \rho_{s} c_{p, s}-a}{h_{v}}+1\right) \tilde{T}_{s}-\frac{k_{s}(1-\phi)}{h_{v}} \frac{\partial^{2} \tilde{T}_{s}}{\partial x^{2}}
$$

and differentiation of Eq. (12) with respect to $x$ results in

$$
\frac{\partial \tilde{T}_{f}}{\partial x}=\left(\frac{s(1-\phi) \rho_{s} c_{p, s}-a}{h_{v}}+1\right) \frac{\partial \tilde{T}_{s}}{\partial x}-\frac{k_{s}(1-\phi)}{h_{v}} \frac{\partial^{3} \tilde{T}_{s}}{\partial x^{3}} .
$$

Equations (12) and (13) are substituted into Eq. (11) which reduces the coupled equations to the third order ordinary differential equation

$$
A_{3} \frac{\partial^{3} \tilde{T}_{s}}{\partial x^{3}}+A_{2} \frac{\partial^{2} \tilde{T}_{s}}{\partial x^{2}}+A_{1} \frac{\partial \tilde{T}_{s}}{\partial x}+A_{0} \tilde{T}_{s}=0
$$

with the coefficients

$$
\begin{gathered}
A_{0}=\frac{h_{v}}{(1-\phi) \rho_{s} c_{p, s}} \frac{h_{v}}{\phi \rho_{f} c_{p, f}}-\left(s-\frac{a-h_{v}}{(1-\phi) \rho_{s} c_{p, s}}\right)\left(s+\frac{h_{v}}{\phi \rho_{f} c_{p, f}}\right), \\
A_{1}=\frac{u_{m}}{\phi}\left(s-\frac{a-h_{v}}{(1-\phi) \rho_{s} c_{p, s}}\right), \\
A_{2}=\frac{k_{s}}{\rho_{s} c_{p, s}}\left(s+\frac{h_{v}}{\phi \rho_{f} c_{p, f}}\right), \\
A_{3}=-\frac{u_{m}}{\phi} \frac{k_{s}}{\rho_{s} c_{p, s}} .
\end{gathered}
$$

The solution of Eq. (14) is

$$
\tilde{T}_{s}(s, x)=\sum_{i=1}^{3} C_{i} \exp \left(\lambda_{i} x\right)
$$

with eigenvalues $\lambda_{i}$ and coefficients $C_{i}$. Substitution of this solution into Eq. (10) yields the fluid temperature in the Laplace domain

$$
\tilde{T}_{f}(s, x)=\sum_{i=1}^{3} C_{i}\left(\frac{s(1-\phi) \rho_{s} c_{p, s}}{h_{v}}+\frac{k_{s}(1-\phi)}{h_{v}} \lambda_{i}^{2}\right) \exp \left(\lambda_{i} x\right) .
$$

The eigenvalues $\lambda_{i}$ are calculated by solving the characteristic equation

$$
A_{3} \lambda_{1-3}^{3}+A_{2} \lambda_{1-3}^{2}+A_{1} \lambda_{1-3}+A_{0}=0
$$

The three boundary conditions Eqs. $7[9]$ and the three unknown coefficients $C_{i}$ form a linear system of equations in the 
Laplace domain that can be expressed as the following matrix form

$$
\left(\begin{array}{ccc}
B_{1} \exp \left(\lambda_{1} L\right) & B_{2} \exp \left(\lambda_{2} L\right) & B_{3} \exp \left(\lambda_{3} L\right) \\
\lambda_{1} \exp \left(\lambda_{1} L\right) & \lambda_{2} \exp \left(\lambda_{2} L\right) & \lambda_{3} \exp \left(\lambda_{3} L\right) \\
\lambda_{1} & \lambda_{2} & \lambda_{3}
\end{array}\right) \cdot\left(\begin{array}{c}
C_{1} \\
C_{2} \\
C_{3}
\end{array}\right)=\left(\begin{array}{c}
0 \\
0 \\
\frac{-1}{k_{s}(1-\phi)}
\end{array}\right)
$$

with

$$
B_{i}=\frac{s(1-\phi) \rho_{s} c_{p, s}}{h_{v}}+\frac{k_{s}(1-\phi)}{h_{v}} \lambda_{i}^{2}
$$

which can be solved either analytically or numerically. This yields an analytical solution in the Laplace domain. However, a transformation back into the time domain has not been attempted. Instead, the algorithm of Gaver and Stehfest [26, 27] is used to numerically perform this operation through

$$
T_{s, f}(t, x)=H_{s, f}(t, x)=\text { real }\left[\frac{\ln 2}{t} \sum_{n=1}^{N=10} V_{n} \tilde{T}_{s, f}\left(s=n \frac{\ln 2}{t}, x\right)\right]
$$

with

$$
V_{n}=(-1)^{N / 2+n} \sum_{m=\frac{n+1}{2}}^{\min (\mathrm{n}, \mathrm{N} / 2)} \frac{m^{N / 2+1}(2 m) !}{(N / 2-m) ! m !(m-1) !(n-m) !(2 m-n) !} .
$$

where the Laplace transformed temperatures are calculated by Eqs. (19) and 20]. When $s=0$ (steady state) and $a=0$ (no heat loss) are used, the calculated temperatures coincide with the solution obtained by Schweikert et al. who solved the same equations for an adiabatic steady state case [18].

The developed model provides the mathematical basis in the Laplace space and allows for a very fast backtransformation into the time domain by the Gaver-Stehfest algorithm. As the heat transfer is solved analytically in the Laplace domain the numerical component of the model is minimal and hence allows for much faster calculations than a conventional finite element approach of porous heat transfer. Furthermore, as the space domain is solved completely analytically, only the time domain is discretized which reduces uncertainties in a numerical procedure. This model exhibiting these characteristics also lends itself to systematic optimization studies that require fast calculation times [28].

\section{Methodology and Experimental Setup}

As the developed model is intended to enable inverse heat transfer analysis, an experimental validation of the calculated impulse responses is required. Additionally, the model is used to highlight the sensitivity of the impulse response with regard to the internal heat transfer between solid and fluid phase. This is also demonstrated by comparing experimental and simulated data. The methodology used to achieve this is shown schematically in Fig. 2. Experimental measurements are conducted using a porous $\mathrm{ZrB}_{2}$ sample. It is very hard to directly measure an experimental impulse 
response due to two major issues: The availability of a heating source that provides an approximate Dirac impulse of heat flux at the surface and the very low expected magnitudes of the impulse response which are in the order of $0.1 \mathrm{mK}$ for the current material. Therefore, an alternative approach is used where the porous sample is subjected to a number of pseudo-random laser pulses that produce higher sample temperatures which result in a good signal to noise ratio of the measurement. These measurements are subsequently used in a non-integer system identification (NISI) which is applied to calculate the experimental impulse response [29-32]. This method has been proven to produce the impulse response of a particular experimental setup and has first been applied to transpiration cooled materials in an investigation of Carbon-Carbon (C/C) samples [23]. Both the modelled and experimental impulse responses are then compared to empirically determine the lateral heat loss constant $a$. Furthermore, the comparison of different mass fluxes gives insight into the internal heat transfer characteristics between solid and fluid. In the following, the experimental setup and the procedure in data reduction are discussed in more detail.

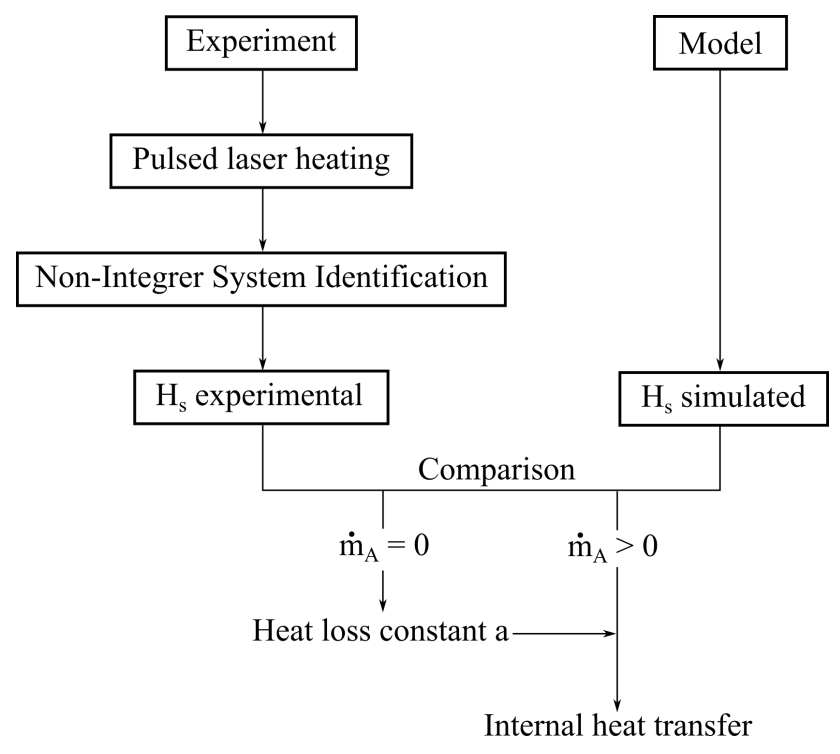

Fig. 2 Methodology for the comparison between experiment and model.

Table 1 Material properties of $\mathrm{ZrB}_{2}[33-35]$.

\begin{tabular}{ll}
\hline \hline$\phi$ & $42 \%$ \\
$k_{s}$ & $41.4 \mathrm{~W} \mathrm{~m}^{-1} \mathrm{~K}^{-1}$ \\
$c_{p, s}$ & $437.6 \mathrm{~J} \mathrm{~kg}^{-1} \mathrm{~K}^{-1}$ \\
$\rho_{s}$ & $6080 \mathrm{~kg} \mathrm{~m}^{-3}$ \\
$K_{D}$ & $2.443 \cdot 10^{-14} \mathrm{~m}^{2}$ \\
$K_{F}$ & $8.855 \cdot 10^{-8} \mathrm{~m}$ \\
$\varepsilon$ & 0.75 \\
\hline \hline
\end{tabular}



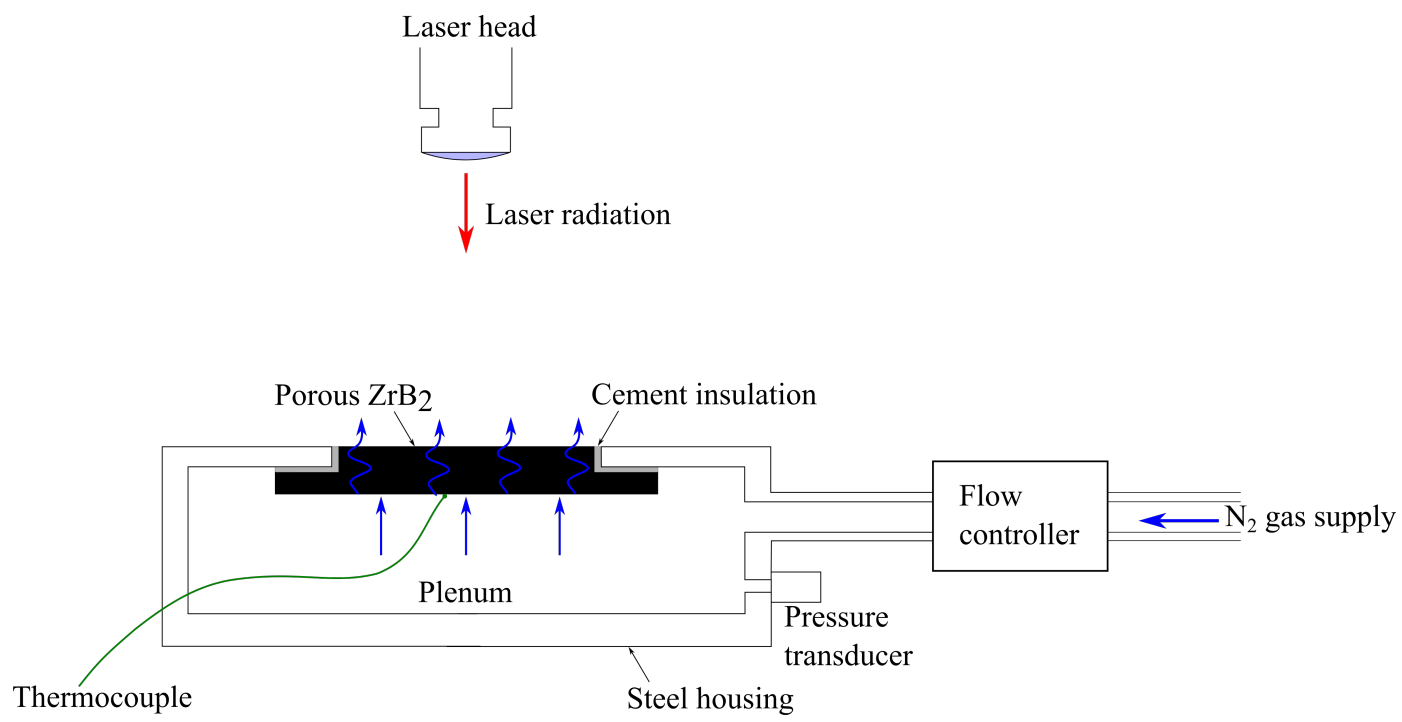

Fig. 3 Schematic cross-sectional view of the experimental setup.

\section{A. Experimental setup}

The experiments reported in this work have been performed with the High Enthalpy Flow Diagnostic Group (HEFDiG) at the Institute of Space Systems (IRS) of the University of Stuttgart. The test article of this investigation is a partially sintered $\mathrm{ZrB}_{2}$ sample with a thickness of $L=5 \mathrm{~mm}$, manufactured by the Department of Materials of Imperial College London [36]. The corresponding material properties are summarised in Table 1] [33- 35]. The schematic setup of the experiment is shown as a cross-sectional view in Fig. 3 and a photograph is presented in Fig. 4 . The sample shape is a square of $32 \times 32 \mathrm{~mm}$ and is mounted within a stainless steel plenum using a layer of cement as sealant and thermal insulation between the two materials. The plenum volume was approximately $2 \cdot 10^{-4} \mathrm{~m}^{3}$.

The sample's surface is heated by a laser (Laserline LDM500-100) with a maximum optical power of $540 \mathrm{~W}$ [31, 37]. The laser heat load is homogeneously distributed over a $68 \times 68 \mathrm{~mm}$ area. The larger heated area is intended to minimize lateral conduction, as the porous sample and the surrounding steel plenum are heated. The backside of the sample is instrumented by a type $\mathrm{K}$ thermocouple which is glued to the porous sample. Both the input power and the thermocouple response are recorded with a LeCroy 24Xs-A oscilloscope while the front temperature is recorded by a FLIR A6751sc SLS thermographic camera. An example image of the thermographic measurement during a laser heating experiment is shown in Fig. 5. The measured surface temperature distribution of the sample is uniform to within $\pm 4 \mathrm{~K}$. The spatial features that are visible in Fig. 5 stem from inhomogeneities of the surface colour and hence lead to a slight change in emissivity (below $7 \%$ ). The spatially even temperature distribution is the basis of the one-dimensional analysis carried out in this work. The gas which is fed into the plenum behind the porous sample is pure nitrogen and is controlled by a Bronkhorst $1 \mathrm{~g} \mathrm{~s}^{-1}$ mass flow controller. The transient pressure signal in the plenum is recorded using both a GEMS 3100 Series ( 0 - 40 bar) and a Kulite ETQ-12-375M-5barA pressure sensor. The coolant mass fluxes used were between 


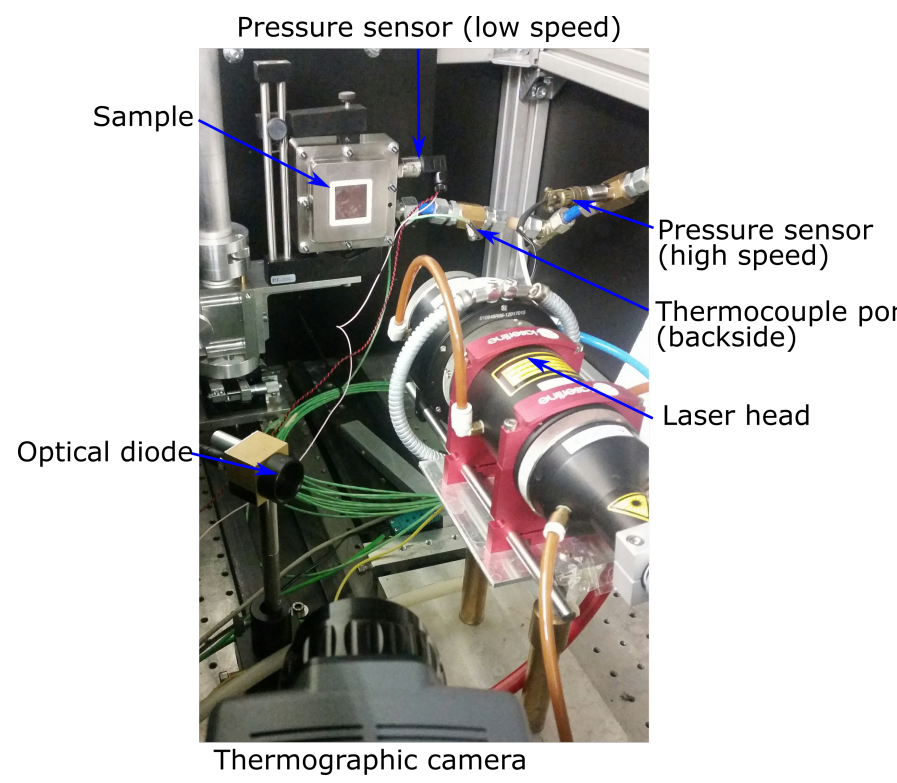

Fig. 4 Overview of the experimental setup at IRS.

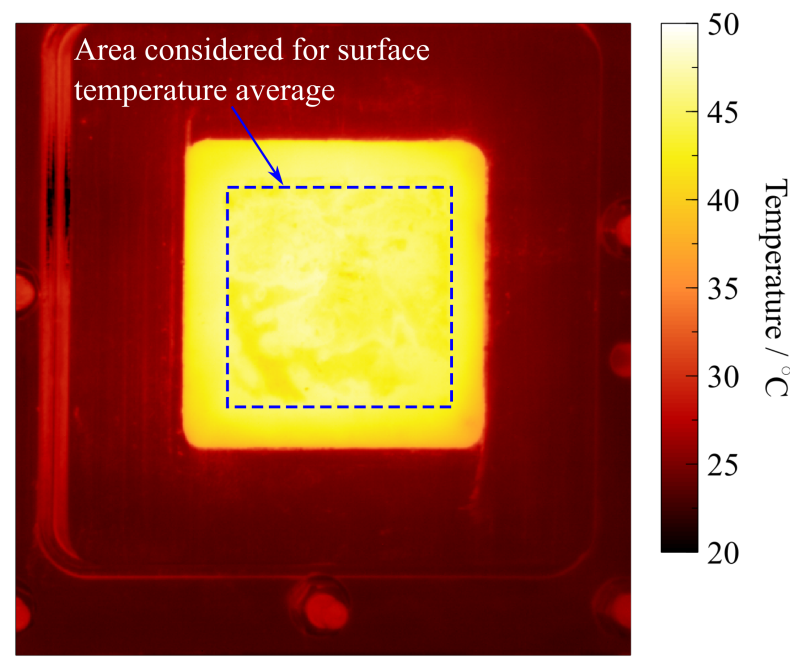

Fig. 5 Example image of a thermography measurement. Area which was used to extract the average surface temperature is shown through dashed lines.

9.8 and $244 \mathrm{~g} \mathrm{~m}^{-2} \mathrm{~s}^{-1}$ which resulted in plenum pressures between 1.2 and 5.8 bar.

\section{B. Non-integer System Identification}

The basic idea of the NISI method is the solution of the heat equation through the use of a system identification [29-32]. The method has originally been derived for the one-dimensional heat equation

$$
\frac{\partial T}{\partial t}(t, x)=\frac{k_{s}}{\rho_{s} c_{p, s}} \frac{\partial^{2} T}{\partial x^{2}}(t, x)
$$

The time domain of this equation is transformed into the Laplace space where a solution is found that relates the surface heat flux and the temperature through the impulse response

$$
H_{s}(t, x)=\frac{\tilde{T}(s, x)}{\tilde{q}(s, x=0)}=\frac{1}{\sqrt{s k_{s} \rho_{s} c_{p, s}}} \sum_{n=0}^{\infty} \frac{(-1)^{n} s^{n / 2} x^{n}}{\left(k_{s} /\left(\rho_{s} c_{p, s}\right)\right)^{n / 2} n !} .
$$

Equation (27) is achieved by a factorization of the $e$-function and derived for the case of semi-infinite boundary conditions [29]. A back-transformation into the time domain results in the characteristic expression

$$
\sum_{m=M_{0}}^{M} \alpha_{m} \mathrm{D}^{m / 2} T(x, t)=\sum_{n=J_{0}}^{J} \beta_{n} \mathrm{D}^{n / 2} \dot{q}(x, t)
$$

with the empirical parameters $\alpha_{m}$ and $\beta_{n}$ linking the non-integer derivatives of heat flux and temperature. These parameter are identified from a calibration measurement where the surface heat flux and the respective temperature are known. This way, the thermal behaviour of a system is identified without assumptions about the magnitude of the 
thermophysical parameters or the respective temperature sensor location. The impulse response of the system can then be determined by calculating the temperature response to a numerical impulse with Eq. (28) using the empirical parameters $\alpha_{m}$ and $\beta_{n}$. Although, the derivation of Eqs. 26] and 277) is carried out for a semi-infinte material, other boundary conditions have been proven to result in the same linking Eq. 28] [30].

The NISI method is chosen as the approach to experimentally determine impulse responses as it has previously been successfully used for cooled surfaces and has no bias toward the thermophysical properties of the experimental setup [23]. It is therefore an ideal candidate to validate the model developed in this work. Furthermore, as the NISI method allows the direct determination of the impulse response of the system, a one to one comparison between experiment and the developed model of this work is possible. In the following, the details of the identification measurements are presented

The calibration heat flux used in Eq. 28 is realized through laser radiation which is absorbed by the sample surface and is calculated by

$$
\dot{q}(t)=\frac{\varepsilon P_{\text {laser }}(t)}{d^{2}}
$$

with the surface emissivity $\varepsilon$ and the laser power, $P_{\text {laser }}$, evenly distributed onto a square area with a length of $d=68 \mathrm{~mm}$. The temperatures reached by the porous material are below $370 \mathrm{~K}$ and radiative heat losses are in the order of $1 \%$ of the calibration heat flux and are therefore neglected. The corresponding measured temperature in Eq. [28] is either the thermocouple data from the backside of the sample or the surface averaged thermographic data. For each of those two cases, the respective impulse response is calculated. The transient shape of the applied heat flux can theoretically be any function. In this work, pseudo-random steps are used as this shape provides information over a wide frequency range [29]. An example of such a calibration experiment is shown in Fig. 6 where the pulsed heat flux profile is shown and the corresponding temperature response at the surface and backside respectively. The coolant mass flux for this particular example was $\dot{m}_{A}=205 \mathrm{~g} \mathrm{~m}^{-2} \mathrm{~s}^{-1}$. The experimental heat flux was filtered in such a way that it was set to zero at times where no heat flux was applied, i. e. between pulses. Additionally, the heat flux and thermocouple data were filtered using a FIR filter to reduce the level of noise. The parameters $\alpha_{m}$ and $\beta_{n}$ in Eq. 28) are determined by a least squares fit using the calibration heat flux and measured temperature. As is evident from Fig. 6 an excellent agreement between the NISI-identification and the measurement data is obtained which gives confidence in the experimentally determined impulse response. The measurement data presented in Fig. 6is the basis for the experimentally determined impulse response which is calculated through Eq. 28. The uncertainty of the temperature measurement was $1.5 \mathrm{~K}$ for the thermocouple and $3 \%$ for the infrared thermography, based on an emissivity uncertainty of $10 \%$. The emissivity also linearly affects the uncertainty of the calibration heat flux. The integral of the temperature measurement, and the calibration heat flux shown in Fig. 6 both scale linearly with the impulse response. The overall uncertainty has been determined using Moffat's method of perturbing the system input parameters, e. g. thermocouple temperature, emissivity, individually by one standard deviation and combining the deviations from the unperturbed solution [38]. The 


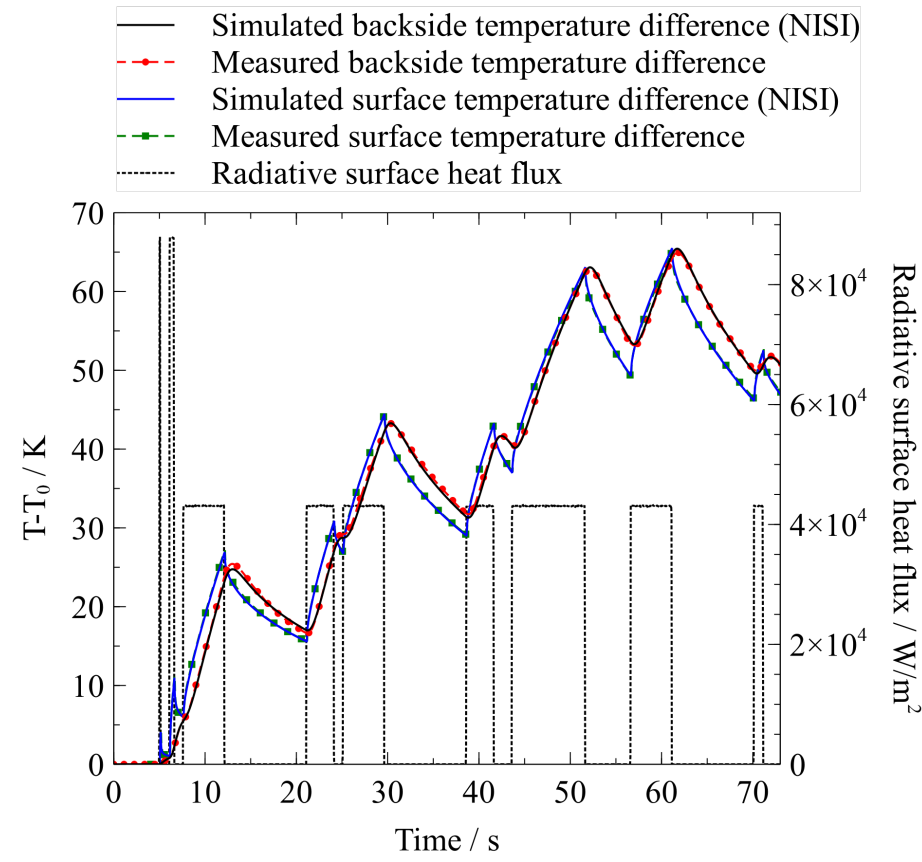

Fig. 6 Example of the NISI identification for the case of $\dot{m}_{A}=205 \mathbf{g ~ m}^{-2} \mathbf{s}^{-1}$.

combined relative uncertainty of the temperature integral and the calibration heat flux applies to the impulse response resulting in $13 \%$ for the surface temperature measurement and in $14 \%$ for the backside temperature measurement.

The next step in the methodology used in this work is the empirical determination of the heat loss constant $a$ of Eq. (6) which is used to model the lateral heat loss in Eq. (4). In order to achieve this, the experimental impulse response for the case of no coolant flow, i. e. $\dot{m}_{A}=0 \mathrm{~g} \mathrm{~m}^{-2} \mathrm{~s}^{-1}$, is used. The respective simulated impulse response using the model detailed in section $\Pi$ is calculated where the parameter $a$ is varied until the best fit between experiment and simulation is obtained. An example of this procedure is shown in Fig. 7 where the experimental and simulated impulse responses for the surface temperature are shown. The simulated impulse response is the best fit obtained with the current model and the error bars refer to the aforementioned uncertainty analysis. This procedure is carried out for both the surface and the backside measurements and the average of the two fits is used. For this work, a loss coefficient value of $a=-2.71 \cdot 10^{4} \mathrm{~W} \mathrm{~m}^{-3} \mathrm{~K}^{-1}$ has been found. Figure 7 also includes impulse responses for fractions and multiples of $a$ showing the sensitivity of the solution to the lateral heat loss.

\section{Results}

This section presents a comparison between the impulse responses determined with the model of this work (denoted as simulated) and those determined experimentally with the help of the NISI method (denoted as experimental). Additionally, the mathematical model is used to put the measurements into a context of a transpiration cooling application. 


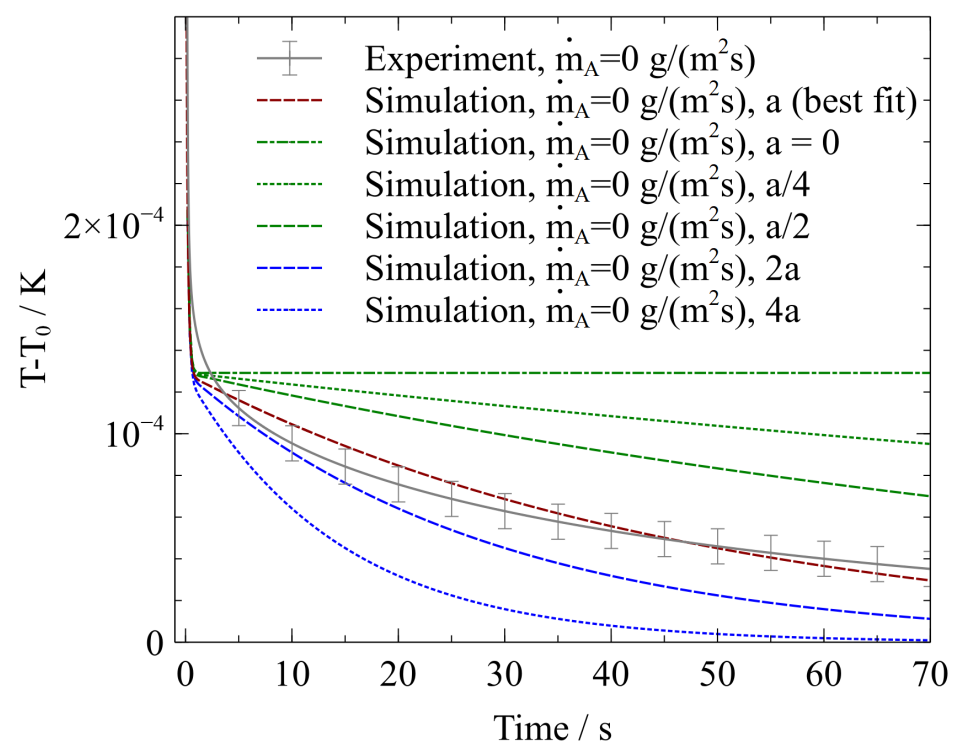

Fig. 7 Example of the fit between experimental and simulated impulse responses used to determine the heat loss parameter $a$.

\section{A. Solid Phase Thermal Impulse Responses}

Figures 8 and 9 present the measured thermal impulse responses of the surface (infrared camera) and backside (thermocouple) that were obtained with the NISI-calibration method for mass fluxes of 0 and $244 \mathrm{~g} \mathrm{~m}^{-2} \mathrm{~s}^{-1}$. The figures contain a direct comparison between experiment and simulation for the maximum and zero blowing cases where the experimental error bars refer to the uncertinaty analysis outlined in section III.B. The influence of the coolant mass flux on the impulse response is evident, as an increased mass flux leads to a lower solid impulse response, i. e. a higher cooling rate. Actually, a higher mass flux also results in a stronger interaction between solid and fluid which is modelled by the volumetric heat transfer coefficient $h_{v}$ in Eqs. (4) and (5) [39]. This effect is also apparent in Figs. 10] and 11] which show a number of different blowing cases for experiment and simulation respectively. As the volumetric heat transfer coefficient of the material is unknown, each simulated impulse response has been fitted to its experimental counterpart by varying the volumetric heat transfer coefficient which is in the order of $5 \cdot 10^{4} \mathrm{~W} \mathrm{~m}^{-3} \mathrm{~K}^{-1}[40]$. The strong dependence of the impulse response on the fluid mass flux clearly shows the importance to incorporate the coupling between solid and fluid phases into the determination of the impulse response. Furthermore, the sensitivity of the impulse response toward the mass flux enables an estimation of the internal heat transfer effectiveness for a given material.

Figure 8 also contains common impulse response models that are classically used for inverse heat transfer problems. The fully adiabatic case is calculated with the current model detailed in section $[$ II with $a=0$, and the semi-infinite case is calculated with Eq. (1) where the thermophysical parameters are adapted to effective values according to the respective 



Fig. 8 Measured and simulated thermal impulse re-Fig. 9 Measured and simulated thermal impulse responses of the solid material at $x=0$. sponses of the solid material at $x=L$.
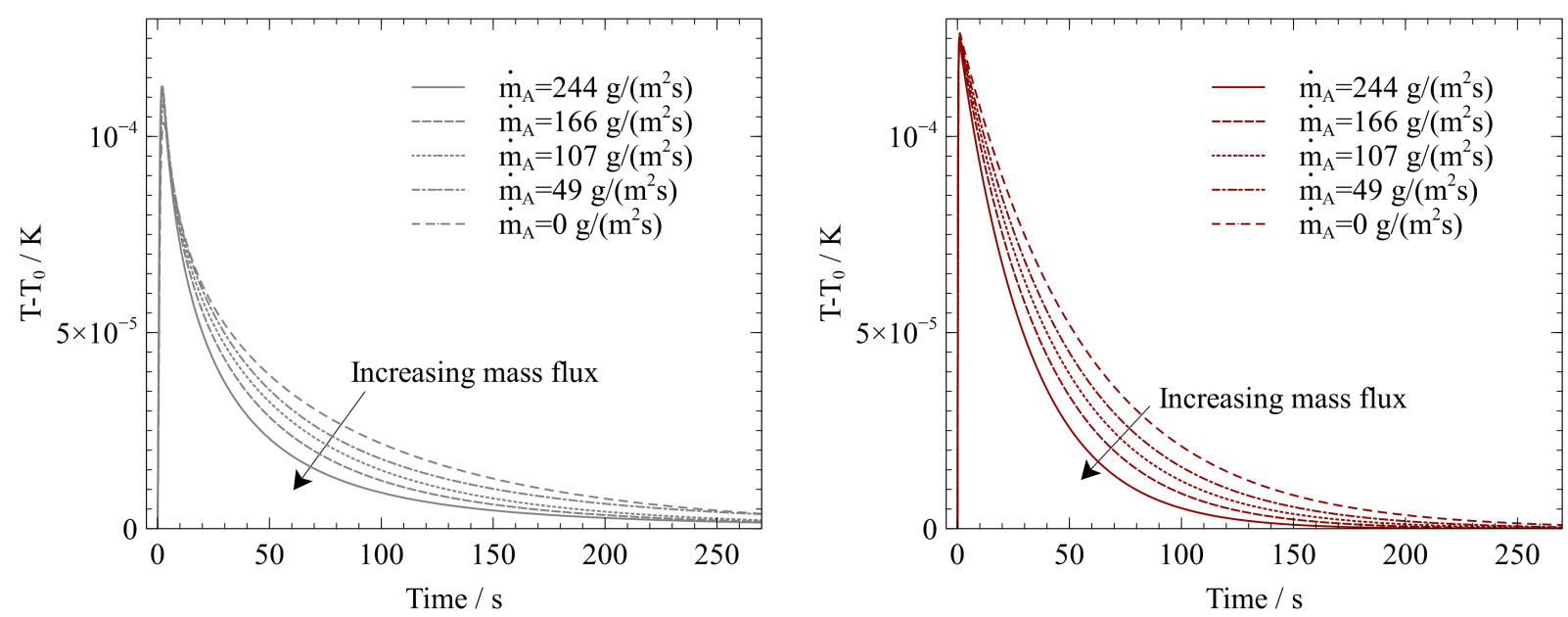

Fig. 10 Measured thermal impulse responses of the Fig. 11 Simulated thermal impulse responses of the solid material at $x=L$. solid material at $x=L$. 
porosity [18, 25]. It is clear from the comparison between these two cases and the experimental measurements that a significant error arises in an inverse heat conduction analysis when the coupling between solid and fluid phases, and the lateral heat loss are not accounted for. The discrepancy is also apparent for the fully adiabatic backside case shown in Fig. 9. The model developed in this work achieves a much better agreement with the measurement data.

Both simulated surface and backside datasets show similar tendencies in absolute magnitude and in the temperature decrease for higher mass fluxes as the respective experimental measurements. However, discrepancies exist: The measured surface impulse responses show a more gradual temperature decrease at short times, whereas the simulation predicts a very steep decrease in the beginning and a delayed onset of the cooling effect. This is most likely due to the finite number of derivatives used in Eq. 28] and the limited frequency resolution of the infrared camera measurement $(30 \mathrm{~Hz})$. In addition, uncertainties in the thermophysical properties contribute to the mismatch. The impulse responses at later times show an excellent agreement below $0.01 \mathrm{mK}$ between experiment and simulation. However, a departure from a linear lateral heat loss behaviour, as modelled through the heat loss coefficient $a$ in Eq. (4), can lead to deviations at later times, as the metal mounting is then more likely to have increased in temperature. The maximum amplitude of the measured backside impulse response is slightly lower than the simulated data. Furthermore, the temperature drop immediately after the maximum is approximately $20 \%$ steeper in the experiment. Both effects suggest a higher heat loss at the backside which could arise through natural convection in the plenum or radiative heat loss. The finite thermocouple response time and uncertainties in the thermophysical parameters could also be a cause for the lower measured amplitude.

Furthermore, the simulation predicts an earlier influence of the cooling, i. e. the impulse responses for different mass fluxes start to spread earlier. A possible explanation is a delayed temperature measurement in the experiment since the thermocouple is enveloped in ceramic glue which adds to the thermal mass of the specific measurement location. As with the surface measurements, the agreement between experiment and simulation is very good (below $0.01 \mathrm{mK}$ ) for later times after $100 \mathrm{~s}$ and the cooling effect is clearly visible.

\section{B. Observations of the coupling of the solid-fluid system from the impulse response behaviour}

The data presented in Figs. 8 and 9 allow some insight into the phenomenological behaviour of the two phases within porous materials. The presented data in this section are purely theoretical and are intended to highlight the physical processes within the material. Porous $\mathrm{ZrB}_{2}$ is simulated with the respective measured thermophysical parameters summarized in Table 1. These simulated impulse responses have been obtained with $a=-2.71 \cdot 10^{4} \mathrm{~W} \mathrm{~m}^{-3} \mathrm{~K}^{-1}$ and $h_{v}=3.5 \cdot 10^{4} \mathrm{~W} \mathrm{~m}^{-3} \mathrm{~K}^{-1}$ which have been determined as the best fit to the measurement data [40].

Simulated solid impulse responses $H_{s}$ are shown in Fig. 12 and the respective fluid impulse responses $H_{f}$ are shown in Fig. 13 for different depths in the material that are spaced equidistantly from each other. Figure 12 shows that the temperature of the solid phase of the material equilibrates within approximately $1 \mathrm{~s}$, due to the high thermal diffusivity 
$k_{s} /\left(\rho_{s} c_{p, s}\right)$ of $\mathrm{ZrB}_{2}$. Heat is lost due to lateral conduction and the heat exchange with the fluid phase. This results in a steady decrease of the solid temperature until the solid phase essentially has been cooled down to the fluid backside temperature $\left(T_{s}=0\right)$. Figure 13 shows that the fluid temperature is lagging behind the solid in both amplitude and phase, a consequence of the finite speed of heat transfer from solid to fluid, i. e. the cause of thermal non-equilibrium. The fluid entering the backside $(x=L)$ is at a temperature of $T_{f}=0$ (imposed by boundary condition (Eq.77). As the fluid is convected through the hot material, it receives more and more heat which leads to an increased temperature at the surface. The larger the internal heat transfer coefficient, the more equilibration between fluid and solid will occur. For $h_{v}=\infty$, the two impulse responses will be identical at a given depth in the material. This behaviour is highlighted in Fig. 14, where fluid and solid impulse responses are shown for various volumetric heat transfer coefficients. With increasing $h_{v}$, the solid and fluid impulse responses keep approaching each other, i. e. get closer to thermal equilibrium. For a transpiration cooling application, the maximum internal cooling performance is achieved for a thermal equilibrium between the two phases.

Figure 15 shows the effect of a varying mass flux on the impulse responses, ignoring that this would also increase $h_{v}$ in a real system [39]. A higher mass flux will increase the thermal non-equilibrium between the phases as the fluid has less time in which to equilibrate since it is convected at a higher speed. However, an increased coolant mass flow also leads to an increased cooling efficiency of the solid, and therefore a lower solid thermal impulse response. The cooling efficiency is further highlighted in Fig. 16] where the integrals of the impulse responses

$$
\int_{0}^{\infty} H_{s}(t) \mathrm{d} t, \quad \text { and } \quad \int_{0}^{\infty} H_{f}(t) \mathrm{d} t
$$
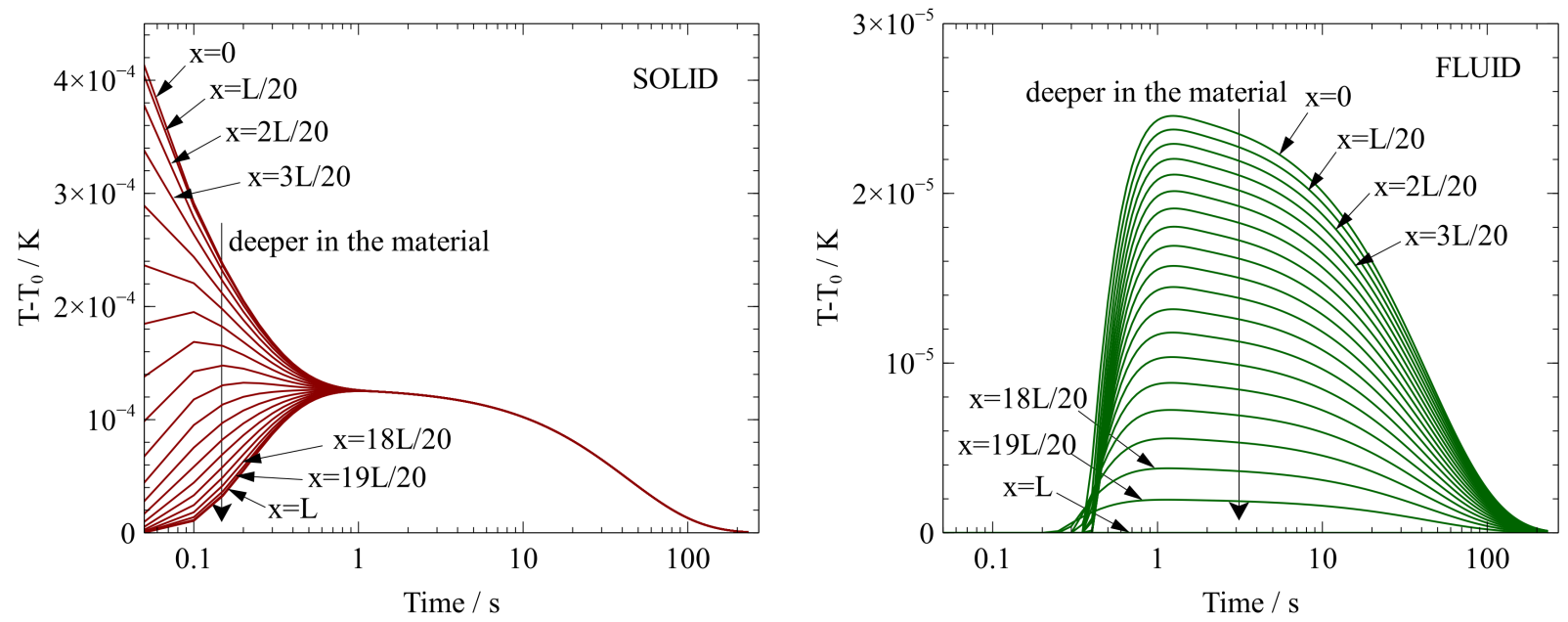

Fig. 12 Thermal impulse responses of the solid mate-Fig. 13 Thermal impulse responses of the fluid at rial at various depths between 0 and $L$ in the material various depths between 0 and $L$ in the material for for $\dot{m}_{A}=186 \mathbf{g ~ s}^{-1} \mathbf{m}^{-2}$. $\dot{m}_{A}=186 \mathbf{g ~ s}^{-1} \mathbf{m}^{-2}$. 



Fig. 14 Fluid (green) and solid (red) impulse responses Fig. 15 Fluid (green) and solid (red) impulse responses at $x=L / 2$ for $\dot{m}_{A}=186 \mathbf{g ~ s}^{-1} \mathbf{m}^{-2}$ and various $h_{v}$.

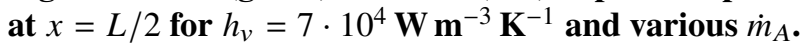



Fig. 16 Integral of fluid (green) and solid (red) impulse responses at $x=L / 2$ for various $\dot{m}_{A}$ and various $h_{v}$ 
are shown for various volumetric heat transfer coefficients and mass flow rates. The integral of an impulse response is indicative of the steady state temperature when the system is exposed to a unit step of heat flux. This is the case since a unit step (Heaviside function) is the integral of a Dirac-impulse. Hence, Fig. 16 essentially provides a first estimate of a steady state behaviour for a transpiration cooled thermal protection system. The steady state temperature of both phases can be extracted as the result in Fig. 16 only has to be multiplied with the net surface heat flux $\dot{q}_{\text {net }}$ to obtain

$$
T_{s, \text { steady }}=\dot{q}_{\text {net }} \int_{0}^{\infty} H_{s}(t) \mathrm{d} t, \quad \text { and } \quad T_{f, \text { steady }}=\dot{q}_{\text {net }} \int_{0}^{\infty} H_{f}(t) \mathrm{d} t .
$$

This highlights a further aspect of the impulse response, as it is very useful in a first stage design process of active cooling systems and provides insight in regimes of varying thermal performance.

The plot shows that the sensitivity of solid phase cooling with respect to mass flux is very high for small mass fluxes since solid-fluid phase equilibration is easier to obtain at lower bulk velocities. Due to this, a state close to thermal equilibrium is present for low mass fluxes which results in a linear behaviour of the solid phase with respect to mass flux. For high mass fluxes, thermal non-equilibrium becomes more prominent which is the reason for a small gain in solid cooling when the mass flux is further increased. However, the volumetric heat transfer coefficient plays an important role in this regime since it promotes thermal equilibrium and hence increases the cooling efficiency, i. e. thermal non-equilibrium is delayed for higher volumetric heat transfer coefficients.

\section{Conclusion}

A solution of the coupled partial differential equations for porous media in thermal non-equilibrium is presented which allows the independent calculation of the thermal impulse response of fluid and solid phase. The impulse response is required to enable inverse heat transfer measurements with transpiration cooled materials. The classical approach of analyzing a porous medium by neglecting the fluid-solid heat exchange fails as porous media impulse responses show a significant sensitivity with regards to the volumetric heat transfer coefficient and the coolant mass flux. Furthermore, this sensitivity enables the analysis of the internal heat transfer effectiveness based on the newly developed model.

A comparison between experimentally measured impulse responses and the respective simulated values is conducted. Experiments are conducted by transient surface heating of a transpiration cooled porous $\mathrm{ZrB}_{2}$ sample through laser radiation. The surface temperature is measured by infrared thermography and the backside temperature is measured using a thermocouple. Both datasets are used in a non-integer system identification which yields the experimentally determined impulse response of surface and backside. The close agreement with average deviations below $10 \%$. between measurement outcome and model simulation shows that the developed model is validated for the analysis of porous candidate materials for transpiration cooling systems. The developed model is found to be suitable for solving the inverse heat conduction problem of porous materials and allows the investigation of transpiration cooling systems in 
more detail.

\section{Acknowledgments}

The help and dedicated attention to detail of Greg King is greatly acknowledged. The authors would like to extend their gratitude towards the entire hypersonics group, high enthalpy flow diagnostics group and the laboratory technical staff. The authors additionally thank Laura Larrimbe, Daniel Glymond and Luc Vandeperre from Imperial College London for providing the material samples, the respective thermophysical properties as well as their advice on the experiment. The funding by EPSRC (Reference: EP/P000878/1) is greatly acknowledged.

\section{References}

[1] Dittert, C., Selzer, M., and Böhrk, H., "Flowfield and Pressure Decay Analysis of Porous Cones," AIAA Journal, Vol. 55, No. 3, 2018, pp. 874-882. doi:10.2514/1.j055298, URL https://doi .org/10.2514/1.J055298

[2] Esser, B., Gülhan, A., Reimer, T., and Petkov, I., "Experimental Verification of Thermal Management Concepts for Space Vehicles," 8th European Symposium on Aerothermodynamics for Space Vehicles, 2015, pp. 1-8. URL http: //elib.dlr.de/97963/

[3] Mohammed Ibrahim, S., Vivek, P., and Reddy, K. P. J., "Experimental Investigation on Transpiration Cooling Effectiveness for Spacecraft Entering Martian Atmosphere,” AIAA Journal, Vol. 54, No. 9, 2018, pp. 2922-2926. doi:10.2514/1.j054757, URL https://doi.org/10.2514/1.J054757

[4] Böhrk, H., Elsässer, H., and Weihs, H., "Flight Data from the faceted TPS on Shefex II," 8th European Symposium on Aerothermodynamics for Space Vehicles, 2015.

[5] Kuhn, M., and Hald, H., "Application of Transpiration Cooling for Hot Structures," RESPACE - Key Technologies for Reusable Space Systems, edited by A. Gülhan, Springer Berlin Heidelberg, Berlin, Heidelberg, 2008, pp. 82-103.

[6] Liu, Y.-Q., Jiang, P., Jin, S.-S., and Sun, J.-G., "Transpiration cooling of a nose cone by various foreign gases,” International Journal of Heat and Mass Transfer, Vol. 53, 2010, pp. 5364-5372.

[7] Basore, K. D., Selzer, M., Wheatley, V., Boyce, R. R., Mee, D. J., Capra, B. R., Kuhn, M., and Brieschenk, S., "Performance comparison of distributed injection methods for hypersonic film-cooling," 20th Australasian Fluid Mechanics Conference, Australasian Fluid Mechanics Society, Perth, WA, 2016. URL https://eprints.qut.edu.au/103495/.

[8] Anderson, J. D., Hypersonic and High-Temperature Gas Dynamics, Second Edition, AIAA Education Series, 2006.

[9] Langener, T., Wolfersdorf, J. V., and Steelant, J., "Experimental Investigations on Transpiration Cooling for Scramjet Applications Using Different Coolants," AIAA Journal, Vol. 49, No. 7, 2011, pp. 1409-1419. doi:10.2514/1.j050698, URL https://doi.org/10.2514/1.J050698 
[10] Tanno, H., Komuro, T., Itoh, K., Kuhn, M., Petkov, I., and Esser, B., “Transpiration cooling experiments in free-piston shock tunnel HIEST," 8th European Workshop on Thermal Protection Systems and Hot Structures, 2016. URL http: //elib.dlr.de/105525/

[11] Kays, W. M., "Heat transfer to the transpired turbulent boundary layer," International Journal of Heat and Mass Transfer, Vol. 15, No. 5, 1972, pp. 1023-1044. URL http://wwW.sciencedirect.com/science/article/pii/0017931072902372

[12] Jiang, P., Yu, L., Sun, J.-G., and Wang, J., "Experimental and numerical investigation of convection heat transfer in transpiration cooling," Applied Thermal Engineering, Vol. 24, 2004, pp. 1271-1289.

[13] Guelhan, A., and Braun, S., "An experimental study on the efficiency of transpiration cooling in laminar and turbulent hypersonic flows," Experiments in Fluids, Vol. 50, No. 3, 2011, pp. 509-525. URL https://doi .org/10 . 1007/s00348-010-0945-6

[14] Otsu, H., Fujita, K., and Ito, T., “Application of the Transpiration Cooling Method for Reentry Vehicles,” No. 0 in Aerospace Sciences Meetings, American Institute of Aeronautics and Astronautics, 2007. doi:10.2514/6.2007-1209, URL https://doi.org/10.2514/6.2007-1209

[15] Ewenz Rocher, M., Hermann, T., McGilvray, M., Ifti, H. S., Hufgard, F., M., E., Meindl, A., Loehle, S., and Zander, F., “Testing a Transpiration Cooled Zirconium-Di-Boride sample in the Plasma Tunnel at IRS," 22nd AIAA International Space Planes and Hypersonics Systems and Technologies Conference, 2019.

[16] Boehrk, H., Piol, O., and Kuhn, M., "Heat Balance of a Transpiration-Cooled Heat Shield," Journal of Thermophysics and Heat Transfer, Vol. 24, No. 3, 2010, pp. 581-588. doi:10.2514/1.47172, URL https://doi.org/10.2514/1.47172

[17] Schweikert, S., von Wolfersdorf, J., Selzer, M., and Hald, H., "Experimental Investigation on Velocity and Temperature Distributions of Turbulent Cross Flows over Transpiration Cooled C/C-Wall Segments," 5th European Conference for Aeronautics and Space Sciences (EUCASS), 2013.

[18] Schweikert, S., von Wolfersdorf, J., Selzer, M., and Hald, H., "Characterization of Actively Cooled Porous C/C Wall Segments According to Pressure Loss and Internal Temperature Distribution," 7th European Workshop on Thermal Protection Systems \& Hot Structures, 2013. URL http://elib.dlr.de/86614/

[19] Dahmen, W., Müller, S., Rom, M., Schweikert, S., Selzer, M., and von Wolfersdorf, J., "Numerical boundary layer investigations of transpiration-cooled turbulent channel flow," International Journal of Heat and Mass Transfer, Vol. 86, 2015, pp. 90-100. URL http://wWw.sciencedirect.com/science/article/pii/S0017931015002483

[20] Beck, J. V., Blackwell, B., and Charles R. St. Clair, J., Inverse Heat Conduction: Ill-Posed Problems, Wiley-Interscience, 1985.

[21] Oldfield, M., "Impulse Response Processing of Transient Heat Transfer Gauge Signals,” Journal of Turbomachinery, Vol. 130, No. 021023, 2008. doi:doi:10.1115/1.2752188. 
[22] Marineau, E., and Hornung, H., "Modeling and Calibration of Fast-Response Coaxial Heat Flux Gages," No. 0 in Aerospace Sciences Meetings, American Institute of Aeronautics and Astronautics, 2009. doi:10.2514/6.2009-737, URL https: //doi.org/10.2514/6.2009-737

[23] Loehle, S., and Fuchs, U., "Heat Flux Calibration Measurement Using the Noninteger System Identification Method for Cooled Surfaces," Journal of Thermophysics and Heat Transfer, Vol. 25, No. 2, 2011, pp. 213-217. doi:10.2514/1.t3631, URL https://doi.org/10.2514/1.T3631

[24] Baghban, M., Shams, Z., and Ebrahimifakhar, A., "Inverse Analysis of a Porous Fin to Estimate Time-Dependent Base Temperature," Journal of Thermophysics and Heat Transfer, Vol. 32, No. 1, 2018, pp. 27-34. doi:10.2514/1.t5004, URL https://doi.org/10.2514/1.T5004

[25] Mayer, B., Gomaa, H., Weigand, B., and Zudin, Y. B., "An analytical model to evaluate heat transfer characteristics in porous media by a periodic quasi steady-state technique," ISROMAC 2012 - 14th International Symposium on Transport Phenomena and Dynamics of Rotating Machinery, 2012.

[26] Gaver, D. P., “Observing Stochastic Processes, and Approximate Transform Inversion,” Operations Research,, Vol. 14, No. 3, 1966, pp. $444-459$.

[27] Stehfest, H., “Algorithm 368: Numerical Inversion of Laplace Transforms [D5]," Commun. ACM, Vol. 13, No. 1, 1970, pp. 47-49. doi:10.1145/361953.361969, URL http://doi . acm.org/10.1145/361953.361969

[28] Hermann, T., Naved, I., and McGilvray, M., "Performance of Transpiration Cooled Heat Shields for Re-entry Vehicles," AIAA Science and Technology Forum and Exposition, San Diego, CA, 2019.

[29] Battaglia, J.-L., Cois, O., Puigsegur, L., and Oustaloup, A., "Solving an inverse heat conduction problem using a noninteger identified model," International Journal of Heat and Mass Transfer, Vol. 44, No. 14, 2001, pp. 2671-2680. URL http://www.sciencedirect.com/science/article/pii/S0017931000003100

[30] Loehle, S., and Fuchs, U., "Theoretical Approach to Surface Heat Flux Distribution Measurement from In-Depth Temperature Sensors," Journal of Thermophysics and Heat Transfer, Vol. 26, No. 2, 2012, pp. 352-356. doi:10.2514/1.t3790, URL https://doi.org/10.2514/1.T3790

[31] Hufgard, F., Loehle, S., Hermann, T., Schweikert, S., McGilvray, M., von Wolfersdorf, J., Steelant, J., and Fasoulas, S., “Analysis of Porous Materials for Transpiration Cooled Heat Flux Sensor Development," International Conference on High-Speed Vehicle Science Technology, CEAS, 2018.

[32] Hufgard, F., Loehle, S., von Wolfersdorf, J., Fasoulas, S., Rocher, M. E., Hermann, T., McGilvray, M., and Steelant, J., “Surface Heat Flux Measurement in Transpiration-Cooled Porous Materials using Plenum Pressure Data," AIAA Science and Technology Forum and Exposition, San Diego, CA, 2019. 
[33] Chase, M. W., of Standards, N. I., and (U.S.), T., NIST-JANAF thermochemical tables, American Chemical Society ; American Institute of Physics for the National Institute of Standards and Technology, [Washington, D.C.]; Woodbury, N.Y., 1998.

[34] Ifti, H. S., Hermann, T., and McGilvray, M., "Flow Characterisation of Transpiring Porous Media for Hypersonic Vehicles," 22nd AIAA International Space Planes and Hypersonics Systems and Technologies Conference, Orlando, Florida, 2018.

[35] Balat-Pichelin, M., Bêche, E., Sciti, D., and Alfano, D., "Emissivity, catalycity and microstructural characterization of ZrB2-SiCfiber based UHTC at high temperature in a non-equilibrium air plasma flow," Ceramics International, Vol. 40, No. 7, Part A, 2014, pp. 9731-9742. URL http://wwW.sciencedirect.com/science/article/pii/S0272884214002582

[36] Wang, J., “Processing and Deformation of ZrB2,” Ph.D. thesis, Department of Materials, Imperial College London, 2012.

[37] Loehle, S., "Derivation of the non-integer system identification method for the adiabatic boundary condition using Laplace transform," International Journal of Heat and Mass Transfer, Vol. 115, 2017, pp. 1144-1149. URL http://www.sciencedirect.com/science/article/pii/S0017931017309389

[38] Moffat, R. J., "Describing the uncertainties in experimental results," Experimental Thermal and Fluid Science, Vol. 1, No. 1, 1988, pp. 3-17. URL http://wWw. sciencedirect.com/science/article/pii/089417778890043X.

[39] Florio, J., Henderson, J. B., Test, F. L., and Hariharan, R., "Characterization of Forced Convection Heat Transfer in Decomposing, Glass-Filled Polymer Composites,” Journal of Composite Materials, Vol. 25, No. 11, 1991, pp. 1515-1539. doi:10.1177/002199839102501108, URL https://doi.org/10.1177/002199839102501108

[40] Hermann, T., McGilvrayy, M., and Ifti, H. S., "Fluid-Solid Heat Exchange in Porous Media for Transpiration Cooling Systems," AIAA Science and Technology Forum and Exposition, San Diego, California, 2019. 